$>$ Le séquençage du génome de l'homme et de la souris a montré que le patrimoine génétique de ces deux espèces comporte environ 30000 gènes. Les phénomènes biologiques que l'on peut étudier dans un organisme ou un tissu résultent d'interactions complexes au sein de cette multitude de gènes. Avant de décrire ces interactions, il faut pouvoir comprendre la fonction de chaque gène. Chez la souris, les lignées congéniques permettent d'introduire un segment chromosomique dans un fonds génétique consanguin déterminé. On peut alors comparer les effets de différents allèles d'un même locus dans un fonds génétique identique ou l'effet d'un même allèle dans différents fonds génétiques. On peut également isoler dans des lignées congéniques différentes possédant le même fonds génétique les gènes contrôlant un caractère génétique complexe, puis combiner ces gènes par des croisements pour en étudier les interactions. Si le segment transféré dans une lignée congénique contient généralement plusieurs centaines de gènes, l'utilisation de marqueurs génétiques permet de réduire ce nombre, ainsi que le nombre de croisements nécessaire. <

\section{Utilisation des lignées congéniques chez la souris}

Xavier Montagutelli, Marie Abitbol

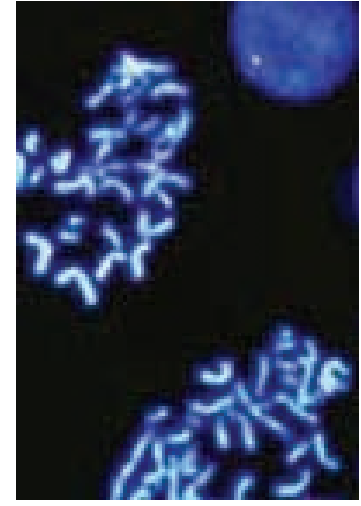

Cependant, les animaux transgéniques sont souvent produits dans des fonds génétiques hybrides qui procurent de meilleurs rendements de transgenèse. Ces animaux possèdent alors un fonds génétique non fixé et non standardisé, qui rend difficiles et peu puissantes les comparaisons entre animaux transgéniques et non transgéniques, et les comparaisons avec les données publiées dans la littérature. II est alors souhaitable de transférer le transgène dans une lignée consanguine en produisant une lignée congénique.

L'introduction d'un allèle provenant d'une lignée (consanguine ou non) dite «donneuse »dans une lignée consanguine dite « receveuse » est réalisée par des croisements en retour répétés entre des souris porteuses de cet allèle et des souris de la lignée receveuse. Considérons le cas d'un transgène que l'on veut transférer de la colonie non consanguine $A$ vers la lignée consanguine $B$ (Figure 1). Une souris hétérozygote pour le transgène est accouplée avec une souris de la lignée B. La moitié de leurs descendants, notés N1, sont hétérozygotes pour le transgène, et identifiés par PCR (polymerase chain reaction) ou Southern blot. Ils sont à leur tour accouplés avec des souris de la lignée B pour pro- 
duire des individus notés N2, parmi lesquels des hétérozygotes seront identifiés puis utilisés pour produire une génération N3, et ainsi de suite jusqu'à l'obtention d'animaux N10. À ce stade, les individus hétérozygotes sont accouplés soit avec des individus de la lignée $B$, pour poursuivre les croisements en retour, soit entre eux, pour produire une colonie dans laquelle le transgène est mis à l'état homozygote. Un protocole comportant au total 20 générations est nécessaire lorsqu'il n'est pas possible d'identifier à chaque génération les individus hétérozygotes, par exemple une mutation récessive dont le gène n'est pas connu [1].

À chaque croisement avec la lignée receveuse, la proportion du génome des individus qui provient de la lignée donneuse est divisée par 2. Elle est ainsi de $50 \%$ chez les individus N1, 25\% chez les N2, $12,5 \%$ chez les N3... Chez les N10, elle atteint la proportion théorique de 0,1\%. Ce calcul, qui considère les gènes comme des entités génétiquement indépendantes dont les allèles se réassortissent au hasard, décrit assez bien ce qui se passe aux locus portés par d'autres chromosomes que celui qui contient le transgène: il est ainsi fort peu probable que les individus portent encore des allèles de la lignée donneuse sur ces chromosomes après dix générations de croisements en retour avec la lignée receveuse. $\varepsilon n$ revanche, ce calcul ne s'applique pas aux locus situés à proximité du transgène. En effet, on sélectionne à chaque génération des individus hétérozygotes pour le transgène. En raison de l'existence de liaisons génétiques, ces animaux sont également hétérozygotes aux locus situés dans le voisinage immédiat du transgène, d'autant plus fréquemment que les locus sont situés à proximité du site d'insertion du transgène. Ainsi, ce n'est pas le transgène seul qui a été transféré lors de la production de la lignée congénique, mais un segment chromosomique contenant le site d'intégration du transgène. Après dix croisements en retour, la taille moyenne de ce segment chromosomique est d'environ 20 centimorgans (cM) ou 40 mégabases $(\mathrm{Mb})$, soit environ 1,5\% du génome. Avec une densité moyenne d'un gène pour $100 \mathrm{~kb}$ à l'échelle du génome entier, ce sont en moyenne quelque 400 gènes qui ont été transférés, certains d'entre eux pouvant jouer un rôle dans le phénotype étudié.

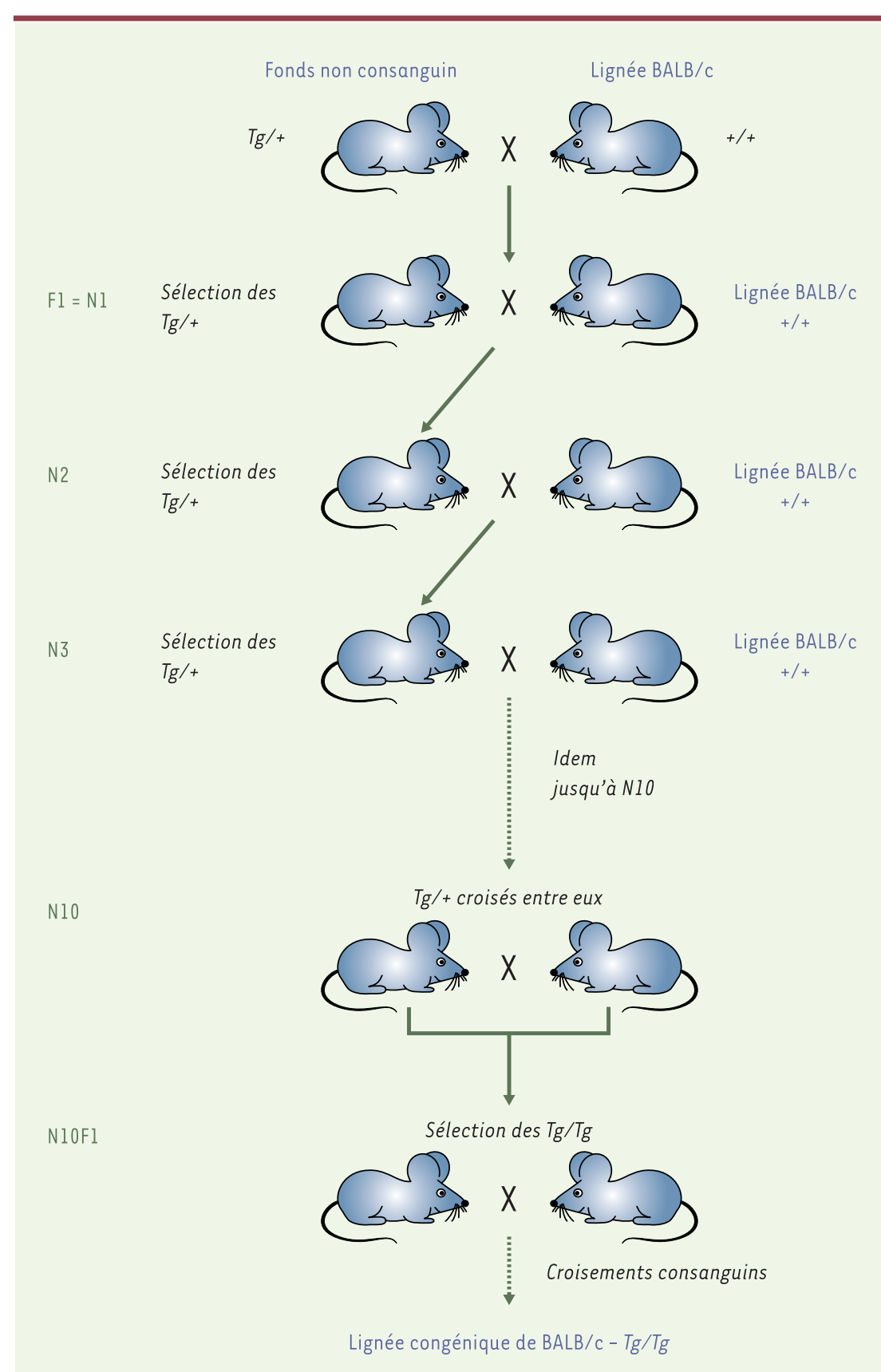

Figure 1. Production d'une lignée congénique pour un transgène. Une souris transgénique est croisée avec une souris de la lignée $B A L B / c$ receveuse. Les descendants transgéniques sont identifiés par PCR (polymerase chain reaction) ou Southern blot et croisés de nouveau avec une souris BALB/c. Cette procédure est renouvelée jusqu'à dix croisements avec la lignée BALB/c. Après 10 générations de croisements en retour, on peut soit réaliser des croisements consanguins dans le but de mettre le transgène à l'état homozygote, soit continuer les croisements en retour pour le maintenir à l'état hétérozygote. 
Pour faire mieux que le hasard et atteindre plus rapidement la proportion théorique de $0,1 \%$ de génome provenant de la lignée donneuse, il faut avoir recours à l'utilisation de marqueurs génétiques polymorphes entre les lignées donneuses et receveuses, tels que les microsatellites. Le protocole dit de speed congenic (Figure 2) a été développé afin d'obtenir en six générations le même résultat qu'avec dix générations de croisements en retour [2, 3]. II repose sur le fait qu'un individu N2, issu du croisement entre un individu N1 et la lignée receveuse, est hétérozygote, en moyenne, à la moitié des locus de son génome et homozygote, en moyenne, à l'autre moitié. Cette proportion moyenne fluctue d'un individu à un autre, de la même façon qu'en tirant 100 fois à pile ou face avec une pièce, on obtient en moyenne 50 fois pile et 50 fois face. Toutefois, on peut observer fréquemment 45 ou 55 fois pile, moins fréquemment 40 ou 60 fois pile, et rarement 35 ou 65 fois pile. Même si ce dernier événement est rare, on peut l'observer à condition de répéter l'expérience plusieurs centaines de fois. De même, si les individus N2 sont homozygotes pour l'allèle porté par la lignée receveuse en moyenne à $50 \%$ des locus, il n'est pas rare d'en trouver qui sont homozygotes à $65 \%$, voire à $70 \%$ des locus (Figure 2). De tels individus sont intéressants, car la proportion de génome de la lignée donneuse qu'ils portent ( $30 \%$ ou $35 \%$ à l'état hétérozygote, soit $15 \%$ à $17,5 \%$ au total) est à mi-chemin entre les proportions moyennes attendues en N2 (25\%) et N3 (12,5\%). Ils permettent de progresser vers l'élimination du génome de la lignée donneuse plus rapidement qu'en comptant sur le réassortiment au hasard des allèles. Pour obtenir ces animaux, une centaine de N2 sont produits, que l'on génotype pour une centaine de microsatellites répartis uniformément le long du génome. On peut s'attendre à obtenir quelques individus homozygotes à $70 \%$ des locus pour l'allèle porté par la lignée receveuse et hétérozygotes pour le transgène. Ils sont utilisés pour produire la génération suivante (N3). La même procédure est répétée pour les N3, qui sont cependant génotypés seulement pour les marqueurs encore à l'état hété- 
rozygote chez leur parent N2. Cette procédure permet d'obtenir, en seulement six générations, le même degré de pureté qu'en dix générations en l'absence de génotypage.

Les marqueurs permettent également de réduire la longueur du segment chromosomique transféré, à condition de connaître la position exacte du locus étudié (le transgène ou une mutation). On utilise deux marqueurs situés de part et d'autre de ce locus et à une distance déterminée par la taille maximale souhaitée pour le segment chromosomique (Figure 3). La stratégie, qui peut être mise en œuvre à n'importe quelle génération de croisement en retour, consiste à produire un nombre suffisant de descendants pour que survienne un événement de recombinaison entre le locus sélectionné et l'un des marqueurs, donnant naissance à un individu hétérozygote pour le transgène et homozygote pour le marqueur: si les marqueurs sont à $3 \mathrm{cM}$ du locus d'intérêt, la probabilité d'obtenir au moins un animal portant un tel génotype sur 100 descendants est supérieure à $95 \%$. Cet individu est utilisé pour produire le croisement en retour suivant. Parmi ses descendants, on en recherche au moins un qui soit hétérozygote au locus et homozygote au deuxième marqueur. Ce faisant, on a réduit en deux générations la longueur de l'intervalle chromosomique transféré à moins de $6 \mathrm{~cm}$.

En combinant les deux approches de sélection par marqueurs, on peut produire des lignées congéniques en 18 mois, avec un degré de pureté prédéterminé. Enfin, si l'on connaît la position du site d'insertion d'un transgène introduit dans une lignée congénique, on peut utiliser des marqueurs moléculaires polymorphes proches de ce site pour identifier plus facilement les individus homozygotes pour le transgène.

\section{Fonds génétique: l'indispensable diversité}

Les lignées congéniques permettent d'étudier les effets biologiques d'un transgène ou d'une mutation dans un fonds génétique consanguin déterminé. $\varepsilon n$ supprimant l'hétérogénéité génétique entre les individus étudiés, elles réduisent les fluctuations phénotypiques incontrôlées qui caractérisent les études effectuées sur des populations naturelles.

Toutefois, analyser la fonction d'un gène sur un fonds génétique consanguin ne permet pas de tirer des conclusions générales en raison des interactions que ce gène entretient avec le reste du génome. En outre, il n'existe pas de fonds génétique consanguin idéal pour toutes les catégories de phénotypes. Un nombre croissant de publications rapporte que le phénotype d'une mutation, spontanée ou issue d'une mutagenèse ciblée ou aléatoire, varie en fonction du fonds génétique sur lequel elle est placée [4-6]. Ainsi, la gravité de plusieurs mutations responsables de polykystose rénale dépend de la lignée consanguine sur laquelle elles se trouvent [7-9]. Un autre exemple célèbre et bien documenté est celui de la mutation Apc ${ }^{\text {Min }}$ (multiple intestinal neoplasia) qui affecte le gène $A p c$ (adenomatous polyposis coli), homologue du gène $A P C$ de l'homme. Les souris de fonds génétique $\mathrm{C} 57 \mathrm{BL} / 6$ hétérozygotes pour cette mutation développent en moyenne 30 tumeurs intestinales, alors que leurs descendants Fl hétérozygotes issus d'un croisement avec la lignée AKR ne présentent en moyenne que 6 polypes $[10,11]$.

Cet effet du fonds génétique, qui est loin d'être exceptionnel, peut être utilisé pour développer de nouveaux modèles de maladies humaines [12]. En effet, en raison

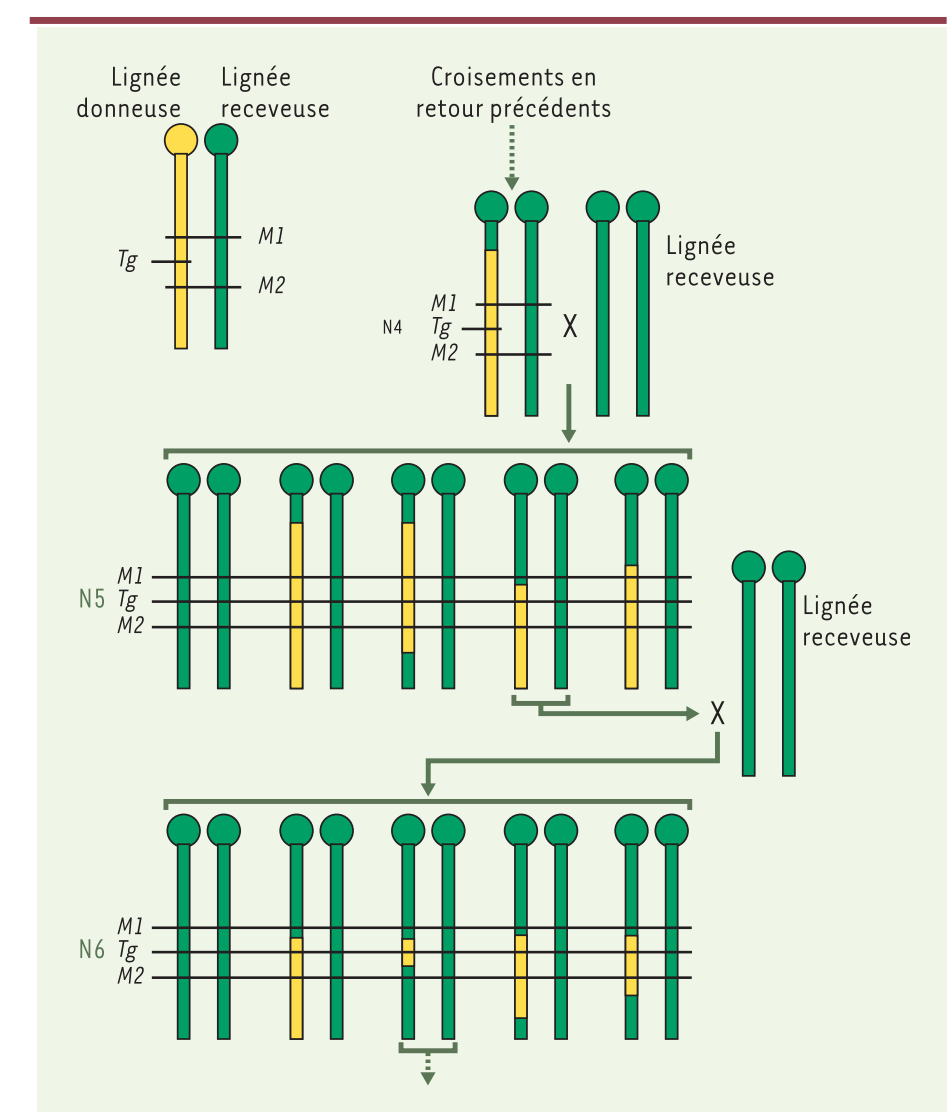

Figure 3. Réduction du segment chromosomique transféré à l'aide de marqueurs génétiques. Cette procédure nécessite que la position du site d'insertion du transgène soit connue et que l'on possède deux marqueurs moléculaires (microsatellites), situés de part et d'autre de ce site, polymorphes entre les lignées donneuse et receveuse (notés MI et M2). Dans cet exemple, plusieurs dizaines de souris $\mathrm{N} 5$ ont été produites pour en identifier au moins une (de préférence un mâle, qui produit plus de descendants qu'une femelle) hétérozygote pour le transgène (allèle jaune) et homozygote à l'un des deux marqueurs pour l'allèle de la lignée receveuse (allèle vert). Elle est utilisée pour produire une génération N6, elle aussi de plusieurs dizaines d'individus, dans laquelle on sélectionne une souris hétérozygote pour le transgène et homozygote pour le deuxième marqueur. 
de l'hétérogénéité génétique, qui est l'une caractéristique de la plupart des maladies génétiques humaines, les patients présentent une gamme de phénotypes parfois très large. Au contraire, une mutation entretenue dans un fonds génétique consanguin induit un phénotype beaucoup plus homogène qui est comparable à celui d'une sous-classe de patients. En transférant la mutation dans différentes lignées consanguines, on peut moduler le phénotype pour le rapprocher de formes cliniques particulières de la maladie humaine homologue. Ainsi, le groupe de $W$. Sly a-t-il montré, dans un modèle d'hémochromatose induit par l'inactivation du gène $\mathrm{Hfe}$ dans des lignées congéniques, que la surcharge hépatique en fer était 3,6 fois supérieure dans le fonds AKR que dans le fonds (57BL/6), une différence proche des variations rencontrées entre des patients porteurs de la mutation C282y du gène HFE humain [13].

II n'existe pas à l'heure actuelle de stratégie permettant de choisir le fonds génétique le plus approprié pour moduler le phénotype d'une mutation dans un sens souhaité. Le projet Mouse phenome database (http: //www.jax.org/phenome), qui a pour but de collecter des informations phénotypiques variées sur plusieurs dizaines de lignées consanguines de souris, pourrait fournir une aide précieuse dans ce choix.

Lorsque deux fonds génétiques associés à des expressions phénotypiques de la mutation contrastées ont été identifiés, des gènes dits «modificateurs », contrôlant ces différences, peuvent être recherchés en utilisant les approches et outils statistiques de la génétique quantitative [1].

\section{Caractères complexes: commençons par faire simple}

Les premières lignées congéniques ont été développées chez la souris, il y a environ 60 ans, par George D. Snell, afin d'isoler les déterminants génétiques de la compatibilité tissulaire [14]. On sait aujourd'hui que ce phénomène biologique est contrôlé par de multiples gènes d'histocompatibilité dont un grand nombre se trouve dans le complexe majeur d'histocompatibilité, les autres étant portés par différents autosomes. Dans son monumental travail de pionnier, G. Snell produisit des lignées congéniques non pas en transférant un segment chromosomique déterminé, mais en sélectionnant, à chaque génération de croisement en retour, des animaux qui rejetaient la greffe d'une tumeur provenant de la lignée receveuse. À force de croisements en retour successifs, il obtint une série de lignées congéniques indépendantes ne différant de la lignée receveuse que pour un seul gène responsable d'un rejet de greffe. II avait ainsi disséqué un phénomène multigénique en une série de caractères monogéniques qu'il avait fixés dans des lignées congéniques distinctes.

L'analyse de caractères complexes chez la souris comporte plusieurs étapes. La première consiste à analyser une population F2 ou N2 issue du croisement entre deux lignées présentant le plus souvent des phénotypes contrastés pour le carac- tère étudié, à la recherche de marqueurs génétiques dont les allèles sont associés à des valeurs phénotypiques différentes. La présence d'un QTL (quantitative trait locus) au voisinage d'un marqueur est suspectée lorsque les animaux porteurs des différents génotypes à ce marqueur $(a / a, a / b$ et $b / b$ pour une F2) présentent un phénotype moyen significativement différent. Les résultats comportent une part d'incertitude, puisqu'aussi bien la présence du QTL que sa localisation dans un intervalle de confiance sont définies en termes de probabilités. Démontrer la présence d'un QTL dans une région chromosomique donnée nécessite de l'isoler dans une lignée congénique.

La production de lignées congéniques pour l'analyse de QTL consiste à transférer non pas un locus, mais un segment chromosomique compris entre deux marqueurs matérialisant les limites de l'intervalle génétique dans lequel se trouve probablement le QTL. Le fait que le phénotype de la lignée congénique diffère significativement de celui de la lignée receveuse permet de confirmer la présence du QTL, en gardant à l'esprit qu'un QTL n'explique qu'une partie de la différence phénotypique entre les lignées parentales. II arrive ainsi fréquemment que la présence du QTL ne puisse pas être confirmée en mesurant le phénotype global de la maladie étudiée (hypertension artérielle, diabète, allergie...), mais seulement en analysant une composante du phénotype global, appelée sous-phénotype (lésions tissulaires, inflammation, concentrations d'immunoglobulines ou de métabolites...). La force des lignées congéniques réside dans la possibilité de produire et d'analyser un nombre illimité d'animaux ayant strictement le même génotype, ce qui accroît considérablement la puissance de détection de différences phénotypiques et la possibilité de démontrer le rôle causal de chaque région chromosomique étudiée. Toutefois, certains caractères résultent d'interactions épistatiques entre QTL très complexes, de sorte que le phénotype peut ne pas être reproductible dans des lignées congéniques où chaque QTL est isolé de ses partenaires.

Le travail exemplaire effectué sur le lupus érythémateux disséminé (LED) par les équipes de $\varepsilon$. Wakeland et L. Morel, de I'Université de Floride, fournit une belle illustration de dissection génétique [15]. Cette maladie auto-immune, caractérisée par la production d'auto-anticorps dirigés contre des antigènes nucléaires, aboutit à une glomérulonéphrite souvent fatale. À partir des lignées C57BL/6, résistante, et NZM2410, sensible, les auteurs ont produit une population F2 et identifié quatre régions du génome contrôlant la sévérité des lésions de néphrite. $\varepsilon$ n isolant chacune des régions dans une lignée congénique de fonds génétique $\mathrm{C} 57 \mathrm{BL} / 6$ et en analysant ces lignées pour des phénotypes immunologiques fins, ils ont pu confirmer la présence de ces quatre QTL et formuler des hypothèses sur leur fonction propre dans le développement de la maladie [16]. Les lignées congéniques ont aussi été utilisées pour bien d'autres phénotypes immunologiques [17]. 
Les lignées congéniques développées pour confirmer l'existence d'un QTL comportent un grand segment chromosomique (souvent plusieurs dizaines de mégabases), trop grand pour envisager d'identifier le gène responsable. On affine la localisation du QTL en fractionnant ce segment en une série de fragments plus petits, partiellement chevauchants et recouvrant l'ensemble de l'intervalle initial, fixés dans autant de lignées dites sous-congéniques (Figure 4). Ce faisant, il est fréquent d'observer que l'effet attribué à un segment chromosomique est dû non pas à un gène, mais à plusieurs gènes génétiquement liés (situés à proximité sur le même chromosome) qui peuvent être isolés dans des lignées sous-congéniques [18-20]. Parfois, ces gènes sont si nombreux et leur effet individuel est si faible que le phénotype s'estompe à mesure que la région est disséquée en plus petits segments [21]. Après avoir permis de disséquer un caractère complexe multigénique en une série de caractères monogéniques, les lignées congéniques peuvent servir à reconstruire la complexité. En croisant entre elles les lignées porteuses des différents QTL, on peut produire des lignées bi- ou
Figure 4. Utilisation de lignées sous-congéniques pour affiner la localisation d'un PTL (quantitative trait locus). A. À partir d'une lignée congénique porteuse d'un grand segment chromosomique, on dérive plusieurs lignées sous-congéniques portant des segments partiellement chevauchants: la lignée congénique (BA) est croisée deux fois avec la lignée receveuse ( $B$ ) pour produire une population de quelques dizaines d'individus qui sont génotypés pour des marqueurs couvrant le segment initial. Les événements de recombinaison chez le parent $\mathrm{Fl}$ ont produit des chromosomes contenant un segment congénique plus court. Les individus qui les portent sont accouplés avec la lignée receveuse et leurs descendants hétérozygotes pour le même segment sont croisés entre eux pour obtenir une lignée souscongénique homozygote. B. L'étude de lignées sous-congéniques permet de confirmer les résultats d'une analyse QTL effectuée sur un croisement, en comparant la composition génétique de chaque lignée avec ses propriétés pour le caractère étudié («effet»). À gauche: les résultats permettent de confirmer l'existence d'un PTL et de réduire l'intervalle dans lequel il doit être recherché. À droite: l'analyse des lignées montre que l'intervalle défini initialement contient en réalité deux PTL liés, combinant leurs effets (aucun sous-intervalle ne permettant à lui seul d'expliquer l'ensemble des résultats, l'effet global est dû à l'effet de l'un et/ou de l'autre (TTL), qui ont pu être isolés dans deux lignées sous-congéniques.

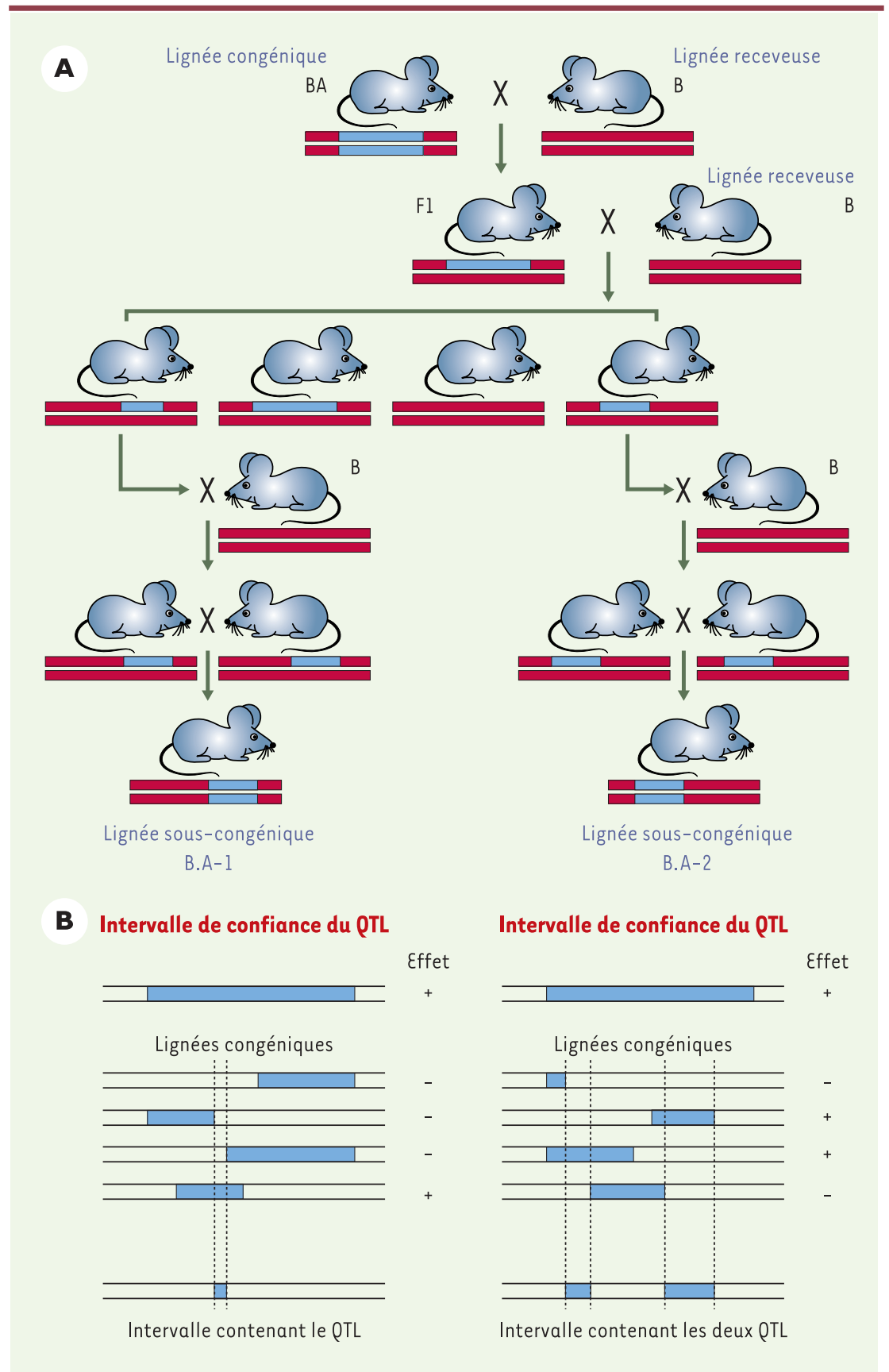


tricongéniques pour étudier les interactions entre ces QTL et identifier les combinaisons de QTL nécessaires et suffisantes pour reproduire le phénotype global étudié. L'exemple le plus remarquable est encore fourni par les travaux de $\varepsilon$. Wakeland et L. Morel sur le LED [22]. Ils ont croisé trois lignées congéniques précédemment décrites selon différentes combinaisons et montré que l'un des QTL est indispensable pour que se développe une glomérulonéphrite lupique, avec un effet aggravant additif des autres QTL.

La stratégie combinatoire s'applique également aux caractères monogéniques. Lorsque des mutations spontanées ou induites sont entretenues sur le même fonds génétique dans des lignées congéniques, deux générations suffisent pour produire des individus double mutants et étudier les interactions épistatiques entre ces mutations. À condition que la taille du segment chromosomique des lignées congéniques soit petite, on dispose ainsi d'un outil d'analyse puissant et inégalé pour l'étude de la complexité des réseaux multigéniques chez les mammifères.

\section{Conclusions}

Les lignées congéniques sont, ces dernières années, passées dans le langage courant de tous les biologistes utilisant les rongeurs de laboratoire. Leur utilisation s'est répandue à mesure qu'a été reconnue la nécessité de maîtriser le fonds génétique des animaux expérimentaux. Elles apportent une contribution essentielle à l'étude du déterminisme des caractères quantitatifs en permettant de tester l'influence d'un segment chromosomique en dehors de tout autre facteur génétique. Leur intérêt continuera de croître lorsque seront identifiées les différences de séquence existant entre les lignées consanguines. II sera alors possible de les produire plus efficacement et de définir précisément leur composition génétique. $\bullet$

\section{SUMMARY}

Applications of congenic strains in the mouse

The sequencing of the human and the mouse genomes has shown that the chromosomes of these two species contain approximately 30000 genes. The biological systems that can be studied in an individual or in a tissue result from complex interactions within this multitude of genes. Before describing these interactions, it is necessary to understand the function of each gene. In the mouse, congenic strains are developed to introduce a chromosomal segment in a given inbred genetic background. One can then compare the biological effects of different alleles at the same locus in the same genetic background or the effect of a given allele in different genetic backgrounds. One can also introduce into different congenic strains with the same genetic background genes which control a complex genetic trait, then combine these genes by appropriate crosses to study their interactions. Although the chromosomal segment transferred into a congenic strain usually contains up to several hundreds of genes, molecular markers can be used to reduce this number as well as the number of crosses required for the development of congenic strains. $\diamond$

\section{RÉFÉRENCES}

1. Panthier JJ, Montagutelli X, Guénet JL. Génétique de la souris. Paris: Belin, 2003: 284 p.

2. Wakeland $\varepsilon$, Morel L, Achey K, et al. Speed congenics : a classic technique in the fast lane (relatively speaking). Immunol Today 1997; 18: 472-7.

3. Markel P, Shu P, Ebeling C, et al. Theoretical and empirical issues for markerassisted breeding of congenic mouse strains. Nat Genet 1997; 17: 280-4.

4. Nadeau JH. Modifier genes in mice and humans. Nat Genet Rev 2001; 2: 165-74.

5. Qiu J, Ogus S, Mounzih K, et al. Leptin-deficient mice backcrossed to BALB/c) genetic background have reduced adiposity, enhanced fertility, normal body temperature, and severe diabetes. Endocrinology 2001; 142: 3421-5.

6. Al-Saktawi K, McLaughlin M, Klugmann M, et al. Genetic background determines phenotypic severity of the Plp rumpshaker mutation. J Neurosci Res 2003; 72: 12-24.

7. laboukova OA, Dushkin H, Beier DR. Genetic analysis of a quantitative trait in a mouse model of polycystic kidney disease. Am J Respir Crit Care Med 1997; $156:$ : $572-7$.

8. Upadhya P, Churchill G, Birkenmeier $\varepsilon H$, et al. Genetic modifiers of polycystic kidney disease in intersubspecific KAT2J mutants. Genomics 1999; 58: 129-37.

9. Guay-Woodford LM, Wright CJ, Walz G, Churchill GA. Quantitative trait loci modulate renal cystic disease severity in the mouse bpk model. J Am Soc Nephrol 2000; 11: 1253-60.

10. Moser AR, Dove WF, Roth KA, Gordon JI. The Min (multiple intestinal neoplasia) mutation: its effect on gut epithelial cell differentiation and interaction with a modifier system. J Cell Biol 1992; 116: 1517-26.

11. Cormier RT, Hong KH, Halberg RB, et al. Secretory phospholipase Pla2g2a confers resistance to intestinal tumorigenesis. Nat Genet 1997; 17: 88-91.

12. Montagutelli $X$. Effect of the genetic background on the phenotype of mouse mutations. J Am Soc Nephrol 2000; 11: S101-5.

13. Fleming RE, Holden CC, Tomatsu S, et al. Mouse strain differences determine severity of iron accumulation in $\mathrm{Hfe}$ knockout model of hereditary hemochromatosis. Proc Natl Acad Sci USA 2001; 98: 2707-11.

14. Guénet JL, Montagutelli X. George D. Snell (1903-1996). Med Sci (Paris) 1997; 13: R7-8.

15. Wakeland EK, Wandstrat AE, Liu K, Morel L. Genetic dissection of systemic lupus erythematosus. Curr Opin Immunol 1999; 11: 701-7.

16. Morel L, Mohan C, Yu Y, et al. Functional dissection of systemic lupus erythematosus using congenic mouse strains. J Immunol 1997; 158: 6019-28.

17. Rogner U, Avner P. Congenic mice: cutting tools for complex immune disorders. Nat Rev Immmunol 2003; 3: 243-52.

18. Bihl F, Brahic M, Bureau JF. Two loci, Tmevp2 and Tmevp3, located on the telomeric region of chromosome 10 , control the persistence of Theiler's virus in the central nervous system of mice. Genetics 1999; 152: 385-92.

19. Morel L, Blenman KR, Croker BP, Wakeland EK. The major murine systemic lupus erythematosis susceptibility locus, Slel, is a cluster of functionally related genes. Proc Natl Acad Sci USA 2001; 98 : 1787-92.

20. Grattan M, Mi QS, Meagher C, Delovitch TL. Congenic mapping of the diabetogenic locus Idd4 to a 5.2-cM region of chromosome 11 in NOD mice. Diabetes 2002; $51: 215-23$.

21. Legare ME, Bartlett II FS, Frankel WN. A major effect PTL determined by multiple genes in epileptic $\varepsilon L$ mice. Genome Res $2000 ; 10: 42-8$.

22. Morel L, Croker BP, Blenman KR, et al. Genetic reconstitution of systemic lupus erythematosus immunopathology with polycongenic murine strains. Proc Natl Acad Sci USA $2000 ; 97: 6670-5$.
TIRÉS À PART

X. Montagutelli 